\section{Rigid Pore Structure from Highly Swollen Polymer Gels}

\section{H. Henning Winter, ${ }^{*, t, \star}$ Griffin Gappert, ${ }^{\dagger}$ and Hisayoshi Ito'}

Department of Chemical Engineering and

Department of Polymer Science and Engineering,

University of Massachusetts at Amherst,

Amherst, Massachusetts 01002

Recei ved November 5, 2001

Revised Manuscript Received February 22, 2002

Innovations in porous materials have driven technological advances in many fiel ds. Catalysis, separations, sound absorption, thermal and electrical insulation, transport through membranes, ${ }^{1}$ and biomedical applications $s^{2,3}$ all benefit from the ability to imbue materials with particular combinations of open pore structure, large specific surface area, and physicochemical properties in a variety of geometries. Techniques to produce porous materials such as sintering, foaming, stretching, leaching, and thermally induced phase separation each enjoy certain advantages within their respective areas of application (typically defined by void fraction, pore size and geometry, specific surface area, material properties, and processing requirements). The final pore structure may be treated in typical ways of physical and chemical surface modification for hydrophilic-to-hydrophobic property change.

The great impact of our new process of creating highly porous materials lies in its environmental friendliness and in its inherent simplicity. Material requirements are minimal. Ideal candidates for this process only require (a) a crystallizable block in the molecule, (b) chemical cross-linking between the molecular chains, and (c) possibility of swelling by an appropriate fluid. The process is termed the "CSX process" for crystallization of swollen, cross-linked polymers. The $\mathrm{P}-\mathrm{T}$ diagram of Figure 1 shows the sequence of processing steps, which leads to the porous structure.

The CSX process starts (state 1 in Figure 1 ) by placing the preshaped, cross-linked polymer specimen into a pressure vessel. At temperatures above crystal melting (state 2 in Figure 1), addition of a suitable solvent transforms the polymer into a swollen gel state (state 3 in Figure 1) in which the cross-linked network provides connectivity. Subsequent crystallization leads to the development of two continuous phases: a solid polymer-rich phase intertwined with a fluid phase (state 4 in Figure 1). After the fluid is removed, a polymer sample with bicontinuous open pore structure remains (state 5 in Figure 1). Rigidity is provided by polymer crystals and by the connectivity of the chemical network that also preserves the original shape of the material. The CSX process transforms a compact solid specimen of polymer (cross-linked and crystallized) into a porous, solid specimen (still cross-linked but newly crystallized) without liquefying the polymer and without external mechanical forces. The forces are supplied internally in the gel by the action of a solvent, which attempts to separate and uncoil the polymer network strands until

* Corresponding author. E-mail winter@ecs.umass.edu.

† Department of Chemical Engineering.

‡ Department of Polymer Science and Engineering.

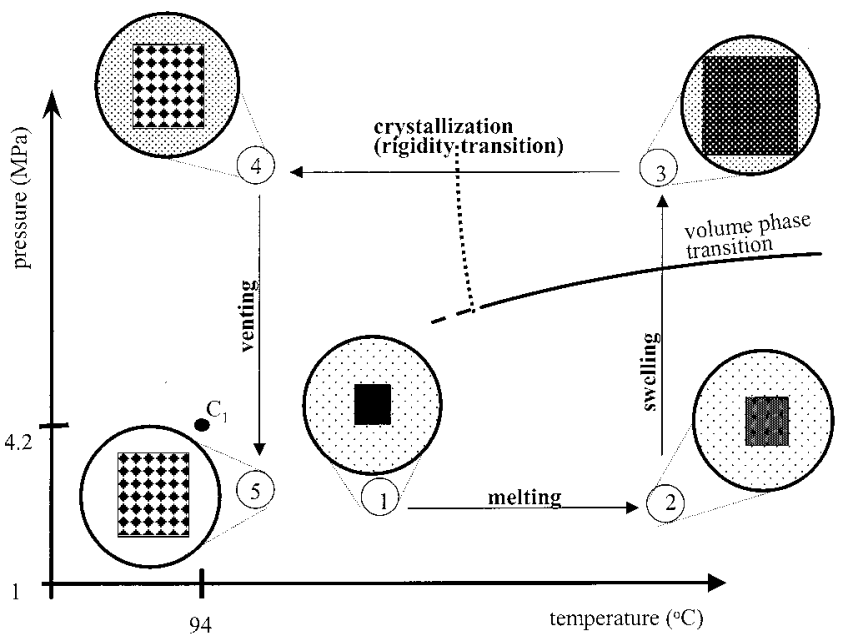

Figure 1. Schematic $P-T$ diagram of the CSX process for generating porous structure through an intermediate gel state. Cross-linked polyethylene with supercritical propane as swelling agent serves as a typical example. The volume-phase transition isopleth and the crystallization temperature (re duced by the presence of the supercritical fluid) for the polyethylene-propane systems were estimated from un-crosslinked PE values in the literature. ${ }^{5}$ The critical point $\left(T_{c}, P_{c}\right)$ of propane is denoted by " $C_{1}$ ". A number and a corresponding cartoon of the specimen indicate each processing step. The cartoons show a specimen (square) in a pressure vessel (circle) at a sequence of states throughout the CSX process: state 1: initial polymer specimen; state 2: amorphous network, rubber; state 3: swollen gel; state 4: bicontinuous structure of polymer and supercritical fluid; state 5: porous polymer after venting of fluid. N ote that the $P-T$ cycle is only drawn schematically; heating and pressurizing can be combined to move directly from state 1 to state 3 , for instance.

tension in the network strands prevents further swelling. For completeness, it should be mentioned that the specimen would return to a compact state with collapsed pores if one would heat it above the melting temperature of the crystallizable block. This possible pore collapse is a special case of the well-known shape-memory effect in cross-linked polymers.

It should be noted that the CSX process is not related to foaming where a small fraction of foaming agent expands its volume severalfold and forms gas bubbles distributed throughout a polymer. The swollen gel of CSX (state 3 in Figure 1) consists mostly of swelling fluid, which does not expand or contract significantly during pore formation. The polymer typically is the minor mass fraction in the gel, and the major mass fraction is the swelling fluid. Also, CSX pore structures differ greatly from the types of periodic structures seen in blown cellular foams.

Supercritical fluids (SCF) are preferred as swelling fluids for CSX for several reasons. SCFs avoid pore collapse during fluid removal, leave no residue at subcritical conditions, and are amenable to complete solvent recovery (zero-emission process). The lack of surface tension in SCF s el iminates destructive capillary forces typical of liquid removal from narrow pores. The tunable solvent character of SCFs can be exploited to completely recover the fluid in high purity. The fluid is readily reused since, at ambient conditions after removal, the fluid loses its ability to solvate and residual contaminants precipitate out of solution. 
We have started to apply this method to commercial polyethylene (HDPE, LLDP), the most common commodity plastic in the world. PE is typically cross-linked by irradiation, by heat-activated radical addition, or by moisture cross-linking after silane grafting. Because of the randomness of the cross-linking reaction, only a fraction of the molecules (gel fraction) actually tether into the network. ${ }^{4}$ The remaining fraction of un-crosslinked molecules (sol fraction) can be substantial.

For the experiments reported here, PE samples were exposed to various amounts of e-beam radiation. The radiation-initiated cross-linking reaction occurs predominantly in the amorphous regions of the solid polyethylene. This implies that the distribution of crosslinks are governed, to a large extent, by the thickness of crystal lamellae, their distribution throughout the sample, and their overall volume fraction. The distribution of cross-links affects the average mesh size in the swollen gel and ultimately the pore structure in the final material. In this way, the crystal morphology prior to cross-linking has far-reaching implications. For radiation cross-linked PE, crystallization conditions and molecular architecture (branch distribution, for instance) become important parameters which will be explored in the future.

We demonstrate the new process using supercritical propane as a swelling fluid. Although polyethylene shows good solubility in many organic solvents at ambient pressure, we chose a SCF because of the previously discussed advantages. The choice of propane goes back to E hrlich and co-workers, ${ }^{5}$ who showed that, at high temperatures and pressures, propane is an effective solvent for un-cross-linked pol yethylene. They al so showed that polyethylene crystallizes readily from such solution and that open-cell structure can be obtained from such solution crystallization. However, the mechanical strength of the resulting structure is low since the crystals are only weakly connected with each other (especially at high pore volume). This problem is overcome in the CSX process by cross-linking the polymer molecules into a chemical network before crystallization.

When exposed to the $\mathrm{P}-\mathrm{T}$ cycle of Figure 1 , a crosslinked polyethylene specimen retains its initial shape but it increases its volume while decreasing its mass and density. The schematic diagram of Figure 1 shows typical data for a polyethylene specimen in supercritical propane. Conditions for the swollen gel (state 3) were chosen as $175^{\circ} \mathrm{C}$ and $62 \mathrm{MPa}$. For pore formation, the gel was crystallized at $85{ }^{\circ} \mathrm{C}$ (state 4). Finally, the swelling fluid was vented by lowering the pressure while remaining temperatures above the critical temperature of the fluid $\left(105^{\circ} \mathrm{C}\right)$ (state 5). Polymer and swelling fluid are chosen such that the melting temperature of the polymer (PE melting at $\mathrm{T}_{\mathrm{m}}=144^{\circ} \mathrm{C}$, ideally) is above the critical temperature of the fluid (propane at $T_{C}=$ $\left.96.7^{\circ} \mathrm{C}\right)$.

The SEM micrograph of a typical porous structure is shown in Figure 2 . The pore sizes strongly vary with cross-linking conditions, polymer properties, and crystallization conditions. Average pore diameters were found to range from $10 \mathrm{~nm}$ to $10 \mu \mathrm{m} .{ }^{6}$ The resulting pore structure has an ultracl ean surface and is ready for use in biomedical applications, for instance. ${ }^{7}$

Experimental evidence suggests that the pore formation process occurs through crystallization of the polymer from a swollen state. Swelling conditions were

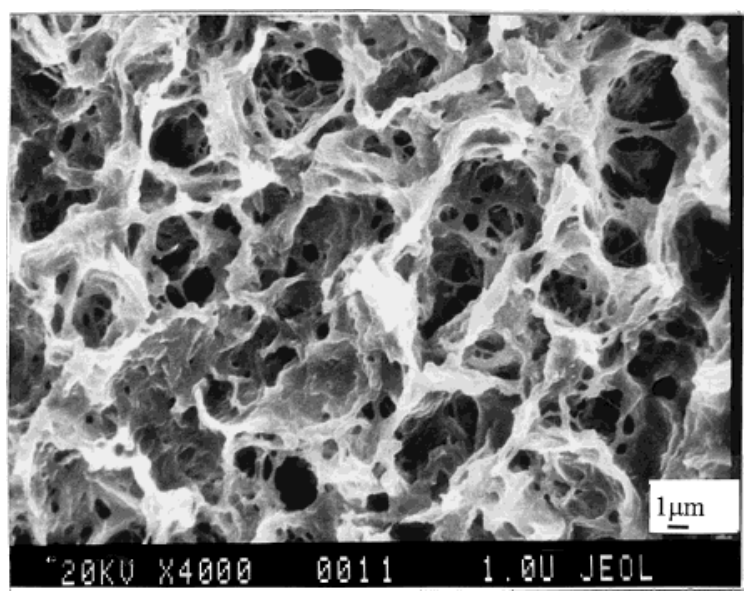

Figure 2. SEM photographs of radiation-cross-linked LLDPE (15.9\% gel content, about $83 \%$ void) after the $\mathrm{P}-\mathrm{T}$ cycle of Figure 1. The SEM involved soaking of the porous specimen in methanol, freezing in liquid nitrogen, fracturing, and sputter-coating with gold; $4000 \times$ magnification.

chosen to be well above the melting temperature of the polyethylene and above the volume phase transition temperature of the gel. Equilibrium swelling is reached when the expansion of the mol ecular chains due to the solvation of monomer units is balanced by the elastic restoring force of the covalently bonded network. This is captured in the Flory-Rehner model. ${ }^{8}$ I ts elastic term can be combined with equations of state to predict the swelling of cross-linked networks in supercritical fluids. ${ }^{9}$ Large degrees of swelling in the gel state are prerequisite for a high void fraction in the final specimen after crystallization. The degree of swelling increases with solvent-monomer interactions, typically at increased temperature and pressure. We have seen that the gel content, which is a measure of cross-link density, is a dominating material parameter for the developing porous structure. If the gel content is too low, the specimen simply dissolves during processing. However, if the gel content is too high, the specimen does not swell sufficiently and no pores develop. Aside from these limiting cases, the relationship between gel content and final pore structure is not yet sufficiently understood. Likewise, the specific volume decreases during crystallization from the swollen state has not been measured directly, although it is believed that the specimen contracts slightly as fluid is expelled from the growing crystalline domains during crystallization.

A specimen loses some mass during the $\mathrm{P}-\mathrm{T}$ cycle since the sol fraction is mobile and gradually diffuses out of the specimen during the swelling (while the remaining sol fraction cocrystallizes with the network chains). For the experiments here, the mass loss was between 16 and 54\%, depending on the degree of crosslinking. Soluble additives present in the material, such as stabilizers or plasticizers, also diffuse into the surrounding fluid. Diffusion times are reduced because of the high mobility of the SCF (swelling fluid). The degree of sol extraction is expected to have significant influence on the final morphology, that is, the structure of crystal lamellae, specific surface area, pore size distribution, and the like. This effect can be kept small by applying only short holding times in the swollen state, by reducing the size of the vessel to that of the swollen specimen, or by extracting untethered molecules prior to processing. In principle, this diffusion phenomenon can also be reversed with the purpose of impregnating 
the swollen specimen with additives dissolved in the swelling fluid. Such additives can be surface modifiers, stabilizers, and functional polymers.

Crystallization has two major functions in the pore formation process. It causes phase separation, and it provides rigidity to the porous structure. Therefore, the dynamics of crystallization as well as by the packing and distribution of crystalline regions need to be controlled. Disruptions of chain regularity (for instance, by branches, cross-links, tacticity variations) are known to reduce the crystallization rate and the crystal fraction in the final state. J unction points of the network are also excluded from crystallization. This would reduce crystallinity at high cross-link density. However, gels as used in the CSX process of Figure 1 require only few cross-links per vol ume. These were found to have nearly no effect on the crystallization. Cooling conditions and crystallization rates have a large influence since, for distributed crystal sizes, the largest, most perfect crystals form at the lowest rate. This will impact the structure of pore walls, which are presumably thicker than individual crystal lamellae. An additional effect is expected from the increased mol ecular mobility in the presence of the supercritical solvent and from the associated melting point depression. All these effects (short-chain branching, mobilization of molecules by supercritical fluid, melting point depression) need to be explored further in the context of pore formation.

Amorphous regions in the semicrystalline polymer are expected to play an important role in the structure formation and in the final properties. After crystallization from the gel state, the specimen consists of (a) pure crystalline polymer, (b) amorphous polymer which is swollen by the supercritical fluid, and (c) pure SCF. The polymer region (amorphous and crystalline) is continuous on molecular level because of the coval ent bonds of the network. The majority of the volume is filled with supercritical fluid, which also forms a continuous phase. During venting, the supercritical fluid gets expelled from the amorphous regions of the polymer (since propane at low pressure is poorly soluble in PE), and the final polymer structure is formed. The vented fluid leaves behind a residuefree, open-pore structure.

One of the challenges in materials design and processing is to achieve and maintain a desired threedimensional shape. Retention of the specified (and often complicated) shape throughout the CSX process allows porosity to be introduced into specimens of well-defined geometry. This unique feature, for instance, is ideal for creating scaffolds of distinct shapes to support directed tissue growth. Cross-linking creates a permanent memory in the specimen, which is retained even through swelling and recrystallization. Therefore, any conventional shaping method (extrusion, injection molding, surface coating) can be used prior to processing, allowing new geometries of porous material to be developed. The variety of shapes possible (thin, thick, round, flat, hollow fibers, coated on surface, prefoamed, filled with particles, layered, etc.) is greater than that allowed by other processes.

The study demonstrates that a cross-linked polyethylene specimen, when exposed to a suitable pressure- temperature cycle in the presence of supercritical propane, increases its size severalfold and develops an open-pore structure. The process is genuine and is expected to be applicable to other semicrystalline polymers which can be cross-linked and swollen. It provides a relatively simple method of obtaining high value added materials from commodity polymers such as polyethylene or from approved biopolymers. When SCF s are employed as the swelling fluid, the process is capable of producing extremely high-purity materials with zero emissions. Current studies focus on the dynamics of pore formation and its effects on final pore structure. Parameters under study are size of the crystallizable chain segment, cross-linking method, cross-link density and homogeneity, SCF character, and $\mathrm{P}-\mathrm{T}$ path of CSX, especially the conditions for swelling and crystal formation. Applications as porous substrate for the adhesion and proliferation of different cell types will also be explored further. Attachment of surfaceactive recognition groups or release agents is envisioned for the future.

Acknowledgment. The National Environmental Institute of the University of Massachusetts (NETI), the MRSEC program at the University of Massachusetts at Amherst (NSF DMR 9809365), and the von Humboldt Foundation gave financial support for this study.

\section{References and Notes}

(1) Pinnau, I. In Encyclopedia of Separation Science; Wilson, I. D., Ed.; Academic Press: San Diego, 2000; Vol. 4, pp 1755-1764. Mark, H. F.; Bikales, N. M.; Overberger, C. G.; Menges, G. In Encyclopedia of Polymer Science and Engineering: Mark, H. F., Ed.; J ohn Wiley \& Sons: New York, 1990; Vol. 9, p 509. Paul, D.; Peinemann, K.-V. In Polymeric Materials Encycl opedia; Salamone, J. C., Ed.; CRC Press: Boca Raton, FL, 1996; Vol. 6, pp 4074-4082. Chiang, C.Y.; Lloyd, D. R. J . Porous Mater. 1996, 2, 273-285.

(2) Suh, J.-K. F.; Matthew, H. W. T. Biomaterials 2000, 21 2589-2598. Glicklis, R.; Shapiro, L.; Agbaria, R.; Merchuk, J. C.; Cohen, S. Biotechnol. Bi oeng. 2000, 67, 344-353. Ma, T.; Li, Y.; Shang-Tian, Y.; Kniss, D. A. Biotechnol. Bioeng. 2000, 70, 606-618. Ma, P. X.; Choi, J .-W. TissueEng. 2001, 7, 23-33.

(3) Lacey, M.; Antonyshyn, O. J . Craniofacial Surg. 1993, 4, 74-78. J anecka, I. P. Arch Otolaryngol Head Neck Surg. 2000, 126, 396-401.

(4) The gel fraction is defined here as mass fraction remaining after $24 \mathrm{~h}$ exposure to Soxhlet extraction with p-xylene at $110{ }^{\circ} \mathrm{C}$.

(5) Bush, P. J .; Pradhan, D.; Ehrlich, P. Macromolecules 1991 24, 1439-1440. Whaley, P. D.; Kulkarni, S.; Winter, H. H.; Stein, R. S.; Ehrlich, P. Polym. Prepr. 1995, 73, 404-405. Whaley, P. D.; Winter, H. H.; Ehrlich, P. Macromolecules 1997, 30, 4882-4886, 4887-4890.

(6) Ito, H.; Gappert, G.; Xia, M.; Fritz, H. G.; Winter, H. H., unpublished experiments.

(7) Preliminary experiments showed $50 \%$ adhesion of HEPG2 liver cells after a $24 \mathrm{~h}$ incubation period under typical conditions: $37^{\circ} \mathrm{C}, 5 \% \mathrm{CO}_{2}$, cell culture medium. The porous $\mathrm{PE}$ was used as is, directly from the CSX process without any further treatment. Sarvat, K.; Gappert, G.; Roberts, S . Winter, H. H., unpublished experiments.

(8) Flory, P. J . Principles of Polymer Chemistry; Cornell University Press: Ithaca, NY, 1953; pp 576-589.

(9) Goel, S. K.; Beckman, E. J . Polymer 1992, 33, 5032-5039.

MA0119225 